\title{
A Whittle's Index Based Approach for QoE Optimization in Wireless Networks
}

\author{
Arjun Anand \\ The University of Texas at Austin \\ Austin, Texas, USA \\ arjun_anand@utexas.edu
}

\author{
Gustavo de Veciana \\ The University of Texas at Austin \\ Austin, Texas, USA \\ gustavo@ece.utexas.edu
}

\begin{abstract}
The design of schedulers to optimize heterogeneous users' Quality of Experience (QoE) remains a challenging and important problem for wireless systems. Our paper( [1]) explores three inter-related aspects of this problem: 1) non-linear relationships between a user's QoE and flow delays; 2) managing load dependent QoE trade-offs among heterogeneous application classes; and 3), striking a good balance between opportunistic scheduling and greedy QoE optimization. To that end we study downlink schedulers which minimize the expected cost modeled by convex functions of flow delays for users with heterogeneous channel rate variations. The essential features of this challenging problem are modeled as a Markov Decision Process to which we apply Whittle's relaxation, which in turn is shown to be indexable. Based on the Whittle's relaxation we develop a new scheduling policy, Opportunistic Delay Based Index Policy (ODIP). We then prove various structural properties for ODIP which result in closed form expressions for Whittle's indices under different scheduler scenarios. Using extensive simulations we show that ODIP scheduler provides a robust means to realize complex QoE trade-offs for a range of system loads.
\end{abstract}

\section{CCS CONCEPTS}

- Mathematics of computing $\rightarrow$ Stochastic control and optimization; - Networks $\rightarrow$ Mobile networks; Wireless access points, base stations and infrastructure; Network algorithms;

\section{KEYWORDS}

Wireless networks, Opportunistic scheduling, Whittle's Index

ACM Reference Format:

Arjun Anand and Gustavo de Veciana. 2018. A Whittle's Index Based Approach for QoE Optimization in Wireless Networks. In SIGMETRICS '18 Abstracts: ACM SIGMETRICS International Conference on Measurement and Modeling of Computer Systems Abstracts, fune 18-22, 2018, Irvine, CA, USA. ACM, New York, NY, USA, 1 page. https://doi.org/10.1145/3219617.3219633

\section{REFERENCES}

[1] Arjun Anand and Gustavo de Veciana. 2018. A Whittle's Index Based Approach for QoE Optimization in Wireless Networks. Proc. ACM Meas. Anal. Comput. Syst. 2, 1, Article 15 (April 2018), 39 pages. https://doi.org/10.1145/3179418
Permission to make digital or hard copies of part or all of this work for personal or classroom use is granted without fee provided that copies are not made or distributed for profit or commercial advantage and that copies bear this notice and the full citation on the first page. Copyrights for third-party components of this work must be honored. For all other uses, contact the owner/author(s).

SIGMETRICS '18 Abstracts, fune 18-22, 2018, Irvine, CA, USA

(c) 2018 Copyright held by the owner/author(s).

ACM ISBN 978-1-4503-5846-0/18/06.

https://doi.org/10.1145/3219617.3219633 